\title{
Quantitative Prediction of Molecular Adsorption: Structure and Binding of Benzene on Coinage Metals
}

\author{
Wei Liu, ${ }^{1,5}$ Friedrich Maaß, ${ }^{2}$ Martin Willenbockel, ${ }^{3}$ Christopher Bronner, ${ }^{2}$ Michael Schulze, ${ }^{2}$ \\ Serguei Soubatch, ${ }^{3}$ F. Stefan Tautz, ${ }^{3,4}$ Petra Tegeder, ${ }^{2}$ and Alexandre Tkatchenko, ${ }^{1, *}$ \\ ${ }^{1}$ Fritz-Haber-Institut der Max-Planck-Gesellschaft, Faradayweg 4-6, 14195 Berlin, Germany \\ ${ }^{2}$ Physikalisch-Chemisches Institut, Ruprecht-Karls-Universität Heidelberg, Im Neuenheimer Feld 253, 69120 Heidelberg, Germany \\ ${ }^{3}$ Peter Grünberg Institut (PGI-3), Forschungszentrum Jülich, 52425 Jülich, Germany \\ ${ }^{4}$ Jülich Aachen Research Alliance (JARA), Fundamentals of Future Information Technology, 52425 Jülich, Germany \\ ${ }^{5}$ Nano Structural Materials Center, School of Materials Science and Engineering, Nanjing University of Science and Technology, \\ Nanjing 210094, Jiangsu, China
}

(Received 24 April 2015; published 17 July 2015)

\begin{abstract}
Interfaces between organic molecules and solid surfaces play a prominent role in heterogeneous catalysis, molecular sensors and switches, light-emitting diodes, and photovoltaics. The properties and the ensuing function of such hybrid interfaces often depend exponentially on molecular adsorption heights and binding strengths, calling for well-established benchmarks of these two quantities. Here we present systematic measurements that enable us to quantify the interaction of benzene with the $\operatorname{Ag}(111)$ coinage metal substrate with unprecedented accuracy $(0.02 \AA$ in the vertical adsorption height and $0.05 \mathrm{eV}$ in the binding strength) by means of normal-incidence $\mathrm{x}$-ray standing waves and temperature-programed desorption techniques. Based on these accurate experimental benchmarks for a prototypical moleculesolid interface, we demonstrate that recently developed first-principles calculations that explicitly account for the nonlocality of electronic exchange and correlation effects are able to determine the structure and stability of benzene on the $\operatorname{Ag}(111)$ surface within experimental error bars. Remarkably, such precise experiments and calculations demonstrate that despite different electronic properties of copper, silver, and gold, the binding strength of benzene is equal on the (111) surface of these three coinage metals. Our results suggest the existence of universal binding energy trends for aromatic molecules on surfaces.
\end{abstract}

Interfaces between organic molecules and inorganic solids have a prominent role in fundamental science and in a wide range of technological applications [1-3]. Such interfaces often demonstrate collective electronic, optical, and vibrational properties that the separate components forming the interface do not exhibit $[4,5]$. These collective effects can be utilized as novel tools in the design of a wide range of technologies. However, the control of properties and functions of hybrid interfaces requires an increasingly reliable level of quantitative understanding of microscopic properties of molecule-surface systems [6-8]. Such fundamental properties are, for example, the vertical adsorption height and the binding strength of a molecule to the solid surface [9]. Many molecules of interest possess one or several aromatic groups with a strong contribution from the ubiquitous $\pi$ interactions to their structure and stability when adsorbed on solid surfaces. To better understand the microscopic underpinnings of such molecules adsorbed on solids and to provide chemically accurate benchmarks for

Published by the American Physical Society under the terms of the Creative Commons Attribution 3.0 License. Further distribution of this work must maintain attribution to the author(s) and the published article's title, journal citation, and DOI. metal- $\pi$ interactions, here we study the adsorption of benzene $(\mathrm{Bz})$ on the $\operatorname{Ag}(111)$ surface as a prototypical model for a molecule-solid interface.

Despite many previous studies of $\mathrm{Bz} / \mathrm{Ag}(111)$ that allowed us to achieve significant insights into this system, the vertical adsorption height $\left(d_{\mathrm{Ads}}\right)$ and the binding strength $\left(E_{\mathrm{Ads}}\right)$ of $\mathrm{Bz} / \mathrm{Ag}(111)$ remain rather uncertain. It is well established that $\mathrm{Bz}$ adsorbs in a planar fashion parallel to the $\operatorname{Ag}(111)$ surface [10-12]. The interaction between $\mathrm{Bz}$ and the $\operatorname{Ag}(111)$ surface can be described by physisorption character-a fact confirmed by inverse photoemission spectroscopy [13], and by high-resolution electron energy loss spectroscopy, which found that the vibrational modes of the molecule upon adsorption on $\operatorname{Ag}(111)$ remain similar to the benzene liquid phase [14]. To our knowledge, there are no direct measurements of $d_{\text {Ads }}$ for $\mathrm{Bz} / \mathrm{Ag}(111)$. Temperature-programed desorption (TPD) studies of $\mathrm{Bz} / \mathrm{Ag}(111)$ yield a desorption temperature of $220 \mathrm{~K}[15,16]$. Depending on the value of the empirical prefactor in the Redhead equation utilized to convert this temperature into $E_{\mathrm{Ads}}$, values ranging from 0.43 to $0.80 \mathrm{eV}$ have been reported in the literature [16-19]. This range of values for $E_{\text {Ads }}$ is almost an order of magnitude larger than the chemical accuracy of $0.04 \mathrm{eV}$ 

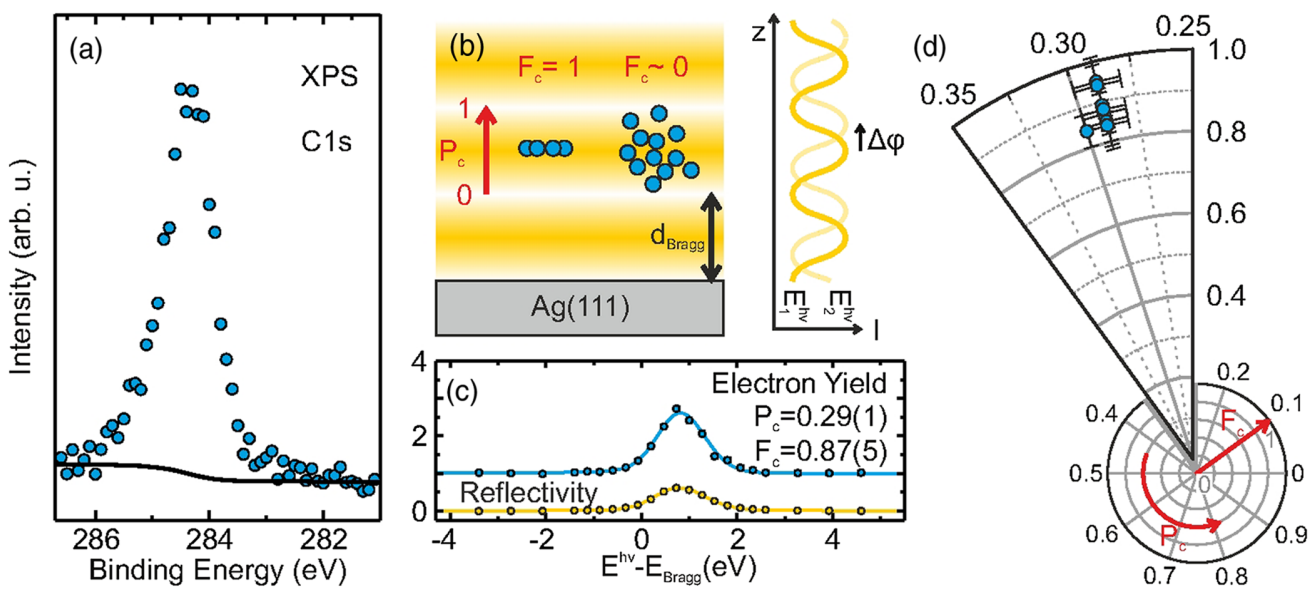

FIG. 1 (color online). The structure of $\mathrm{Bz}$ on $\mathrm{Ag}(111)$ as measured by NIXSW. (a) C1s x-ray photoelectron spectroscopy spectrum of $\mathrm{Bz} / \mathrm{Ag}(111)$ with a single component at $284.3 \mathrm{eV}$, recorded with $E^{h \nu}=2620 \mathrm{eV}$. The area of this carbon contribution is used to determine the electron yield in the NIXSW analysis. (b) Schematic of the NIXSW experiment. The x-ray standing wave field is shown as yellow color gradient, dark yellow meaning high x-ray intensity, and as an intensity curve $I(z)$ on the right. Photoemitters are shown in blue for two situations: a sharp distribution with coherent fraction $F_{c}=1$ on the left, and a homogeneous distribution with $F_{c} \approx 0$ on the right. The coherent position $\left(P_{c}\right)$, indicated by the red arrow, runs from 0 to 1 between two consecutive Bragg planes of the substrate. As the photon energy is tuned through the Bragg condition, the nodes of the standing wave field shift by half a lattice constant, as indicated by the intensity $I(z)$. (c) Blue dots: normalized electron yield of a NIXSW experiment for Bz/Ag(111). According to dynamical scattering theory, the normalized electron yield varies between 0 and 4 . Yellow dots: corresponding reflectivity curve. Lines are the fits obtained with the Torricelli program [25,26] from which $P_{c}$ and $F_{c}$ have been determined. (d) Polar diagram of the NIXSW result, with $P_{c}$ as polar angle and $F_{c}$ as radius. The high $F_{c}(\lesssim 1)$ indicates a flat adsorption geometry of Bz/Ag(111) [cf. left part of panel (b)].

required for a truly predictive description of molecular systems. This uncertainty for a fundamental moleculemetal system is particularly deleterious for the development of new theoretical methods, which are now becoming increasingly more accurate [20]. In this context, it is desirable to achieve a new level of understanding and precisely quantify the interaction of $\mathrm{Bz}$ with $\mathrm{Ag}(111)$.

We start with the determination of the adsorption structure of $\mathrm{Bz} / \mathrm{Ag}(111)$ by utilizing normal-incidence $\mathrm{x}$-ray standing wave (NIXSW) measurements, shown in Fig. 1. On crystalline samples, NIXSW employs the interference of an X-ray beam with its Bragg reflected wave to form a standing wave field extending above the sample surface. Such spatially modulated $x$-ray intensity allows for a chemically sensitive, precise measurement of molecular vertical adsorption distances $[21,22]$. The high coherent fraction $\left(F_{c}\right)$ for $\mathrm{Bz} / \mathrm{Ag}(111)$ in Fig. 1(d) - the NIXSW parameter reflecting the vertical distribution of scatterers-clearly indicates a flat adsorption geometry. The small $10 \%$ deviation of $F_{c}$ from unity stems from a combination of zero-point and temperature-induced vibrations and a small fraction of benzene molecules adsorbed at surface defects, as a detailed investigation of a similar molecule has already shown [23]. Furthermore, the adsorption height can be directly calculated from the NIXSW coherent position $\left(P_{c}\right)$, yielding $d_{\text {Ads }}$ of $3.04 \pm 0.02 \AA$ and suggesting a physisorption-type of binding. The given statistical error was derived from a systematic Monte Carlo based error analysis [24].

We now proceed to determine the adsorption energy of a single $\mathrm{Bz}$ molecule on $\mathrm{Ag}(111)$ by carrying out TPD measurements for a wide range of initial coverages in the submonolayer regime, summarized in Fig. 2. Such coverage-dependent measurements allow us to utilize the so-called complete analysis $[27,28]$ to determine the desorption energy from the TPD spectra based on the PolanyiWigner equation [29]. This procedure does not require any prior assumptions about preexponential factors for desorption. Using such complete analysis, we determined an adsorption energy of $0.68 \pm 0.05 \mathrm{eV}$ for $\mathrm{Bz}$ on $\mathrm{Ag}(111)$ in the limit of vanishing surface coverage. This value already includes the conversion from the measured desorption energy at finite temperature to the adsorption energy at $0 \mathrm{~K}$ by adding $3 / 2 k_{B} T_{\text {des }}(\approx 0.03 \mathrm{eV})$, where $k_{B}$ is the Boltzmann constant and $T_{\text {des }}$ is the desorption temperature [18,30,31]. Surprisingly, the determined magnitude of $E_{\text {Ads }}$ for $\mathrm{Bz} / \mathrm{Ag}(111)$ is remarkably close to the recently computed cohesive energy of one benzene molecule in the benzene crystal [32]. Even more remarkable is the fact that the values of $E_{\mathrm{Ads}}$ for $\mathrm{Bz}$ on $\mathrm{Cu}(111), \mathrm{Ag}(111)$, and $\mathrm{Au}(111)$ determined in our study using the same TPD analysis agree within the measurement error bar $( \pm 0.05 \mathrm{eV})$. The corresponding adsorption energies for $\mathrm{Bz} / \mathrm{Cu}(111)$ and $\mathrm{Bz} / \mathrm{Au}(111)$ are $0.69 \pm 0.04 \mathrm{eV}$ and $0.65 \pm 0.03 \mathrm{eV}$, respectively. This suggests that there must be a simple rule of thumb for predicting the strength of $\pi$ interactions at equilibrium in extended systems. This finding will be further discussed below using first-principles calculations.

Having obtained precise experimental benchmarks of $d_{\mathrm{Ads}}=3.04 \pm 0.02 \AA$ and $E_{\mathrm{Ads}}=0.68 \pm 0.05 \mathrm{eV}$, we now turn to the question of whether state-of-the-art 

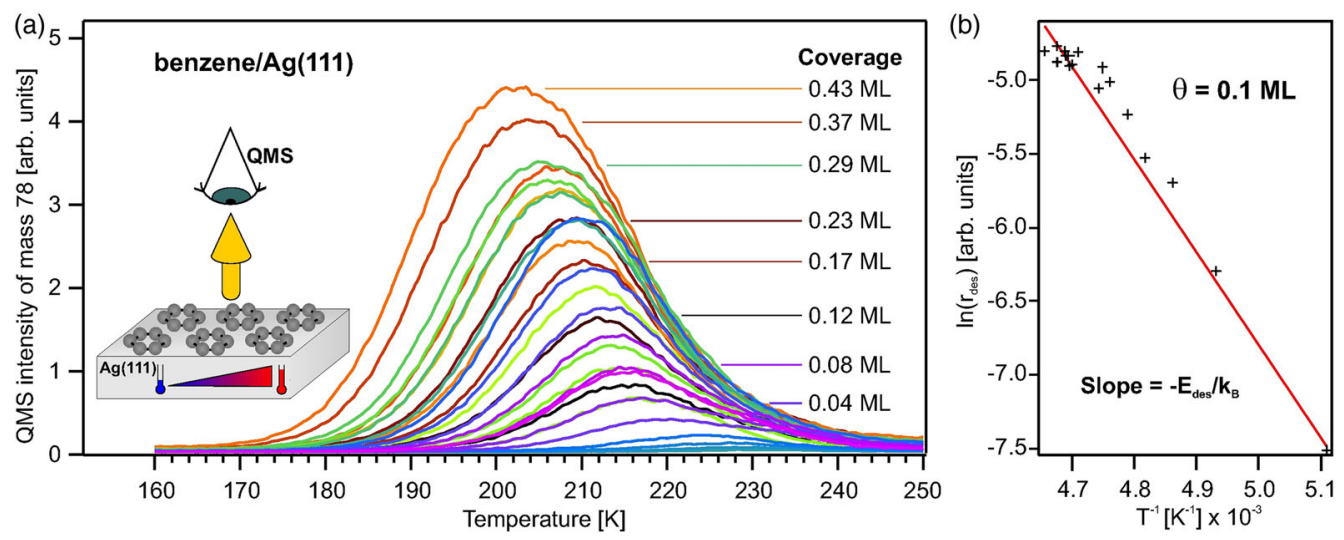

FIG. 2 (color online). The desorption energy of benzene on $\operatorname{Ag}(111)$ as measured by TPD. (a) TPD spectra of benzene adsorbed on $\operatorname{Ag}(111)$ for different initial coverages recorded with a linear heating rate of $1 \mathrm{~K} / \mathrm{s}$ at the mass of the parent ion 78 amu. (b) The logarithm of the desorption rate (in arbitrary units) as a function of the inverse temperature at an exemplary coverage of $\theta=0.1$ ML. The slope $\left(-E_{\mathrm{des}} / k_{B}\right)$ of the line yields the desorption energy $\left(E_{\mathrm{des}}\right)$ for that coverage.

electronic structure calculations are able to achieve quantitative accuracy for the $\mathrm{Bz} / \mathrm{Ag}(111)$ system. We stress that it is a rather ambitious goal to capture all the relevant effects required to describe correctly the binding of molecules on metals [33]. The sheer size of the system and its metallic nature prohibits the use of the well-established hierarchy of quantum-chemical methods for solving the Schrödinger equation. In addition, solids in general, and metals in particular, are characterized by strong dielectric screening within the extended bulk. This makes simple and widely employed pairwise treatments of van der Waals (vdW) interactions inapplicable to molecule-metal systems $[20,33]$. To complicate matters further, even for molecules located at large vdW distances from surfaces, significant interface polarization effects arise, which are accompanied by a strong renormalization of molecular electronic levels [34]. At equilibrium separations, the binding becomes even more complex, with a subtle interplay between Pauli repulsion, wave function hybridization, electrostatics, and $\mathrm{vdW}$ interactions dictating the structure and binding strength between molecules and metals. In this regard, density-functional theory (DFT) has been the workhorse method for studying molecule-metal systems [20]. Despite the increasing success of DFT when applied to adsorption systems, one has to be aware of its two main problems: (i) the self-interaction error in the exchange energy resulting in wrong electronic energy levels and imperfect electrostatics, and (ii) the lack of long-range vdW correlation interactions. Here we directly address these two issues by (i) using a hybrid DFT-HSE exchange-correlation (XC) functional $[35,36]$ that cures a large part of the self-interaction error, and (ii) including many-body vdW correlations with the recently developed DFT + MBD method $[37,38]$.

Our first-principles calculations for the binding-energy curve of benzene on $\operatorname{Ag}(111)$ are shown in Fig. 3. All calculations have been carried out with the FHI-aims code [39], with additional calculation details reported in the
Supplemental Material [40]. The relativistic effects were treated by using scaled zeroth-order regular approximation [41]. In order to demonstrate the importance of manyelectron XC effects, we present three different binding curves that allow us to disentangle the contribution of different terms to the adsorption energy. The comparison between PBE + MBD and HSE + MBD calculations demonstrates the crucial reduction of the self-interaction error when using the HSE XC functional, and the ensuing improvement of static electron density and electrostatic interactions. This can be clearly explained by analyzing the permanent quadrupole moment of benzene at PBE and

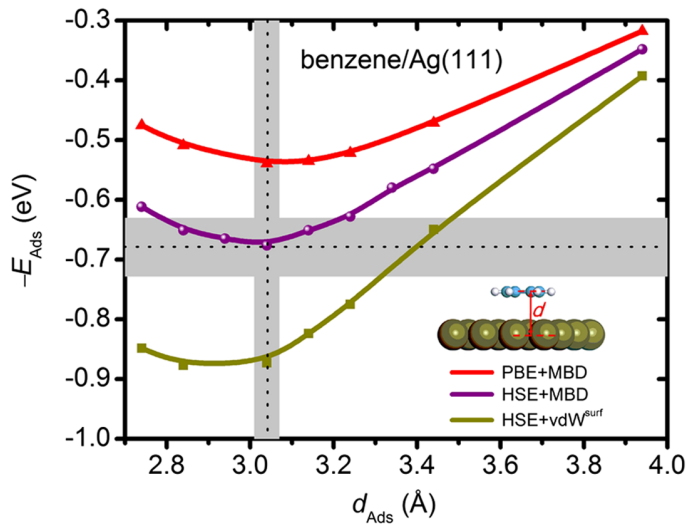

FIG. 3 (color online). First-principles calculations for benzene on $\operatorname{Ag}(111)$. Binding energy $E_{\text {ads }}$ as a function of vertical distance $d_{\text {Ads }}$ of benzene molecule center of mass from $\mathrm{Ag}(111)$. Calculations were carried out using three methods: semilocal Perdew-Burke-Ernzerhof (PBE) [42] functional with many-body dispersion (MBD) [37,38] interactions, or hybrid Heyd-ScuseriaErnzerhof (HSE) functional [35,36] with either vdW ${ }^{\text {surf }}$ [17] or MBD methods for including vdW interactions. Zero-point vibrational energy is included in all binding curves, calculated using a quasiharmonic approximation. The dotted lines indicate experimental equilibrium values of $d_{\mathrm{Ads}}$ and $E_{\mathrm{Ads}}$ with shaded regions corresponding to the associated error bars. 
HSE levels of theory. The PBE calculation yields a value of $6.10 \mathrm{ebohr}^{2}$, while HSE improves this prediction to $6.42 \mathrm{ebohr}^{2}$, being in excellent agreement with the value of $6.42 e^{e b o h r^{2}}$ obtained with the "gold standard" coupledcluster method.

To highlight the importance of collective electronic correlation effects in the interaction of benzene with $\operatorname{Ag}(111)$, we proceed to analyze the $\mathrm{HSE}+\mathrm{vdW}^{\text {surf }}$ and HSE + MBD calculation results in Fig. 3. The first method accurately includes the screening of the $\mathrm{vdW}$ interaction inside the extended metal bulk by utilizing the experimental dielectric function in the determination of the "atom-ina-bulk" vdW parameters [17]. The MBD method goes significantly beyond the $\mathrm{vdW}^{\text {surf }}$ ansatz and treats the collective correlations within the molecule-solid system to all orders of perturbation theory [37,38]. The large reduction in binding at the HSE + MBD level when compared with HSE + VDW ${ }^{\text {surf }}$ stems from significant interface polarization redistribution due to many-body correlation effects. In fact, the polarizability of the adsorbed benzenemetal complex calculated with the MBD method differs significantly from the sum of polarizabilities of isolated benzene and $\operatorname{Ag}(111)$ surface moieties. The perpendicular component of the polarizability increases by $5.8 \mathrm{bohr}^{3}$, while the in-plane polarizability is reduced by 13.7 bohr $^{3}$. This change in polarizability components results in less effective coupling between the in-plane fluctuations of the benzene molecule and $\operatorname{Ag}(111)$ surface plasmons, leading to a concomitantly smaller vdW attraction. The significant polarization redistribution found in our calculations for a prototypical physisorbed system provides an interesting motivation for future experimental studies of local response properties of molecules adsorbed on solid surfaces.

The accurate inclusion of collective nonlocal XC effects in the HSE + MBD approach leads to excellent agreement of both $d_{\mathrm{Ads}}$ and $E_{\mathrm{Ads}}$ with our experimental measurements. Notably, both calculated quantities lie well within the systematic experimental uncertainties. To generalize our findings, further DFT + MBD calculations were carried out for benzene adsorbed on $\mathrm{Cu}(111)$ and $\mathrm{Au}(111)$ surfaces. In agreement with TPD results, we find almost degenerate adsorption energies (within $0.07 \mathrm{eV}$ with $\mathrm{PBE}+\mathrm{MBD}$ and $0.11 \mathrm{eV}$ with HSE + MBD) for benzene on the three coinage metal surfaces. Both values are within the accuracy of the approximate DFT functionals. Interestingly, the adsorption distances differ significantly for the three metals. The binding of $\mathrm{Bz}$ on coinage metals is driven by an interplay between Pauli repulsion (stemming from the antisymmetry of the total molecule-surface wave function), attractive vdW interactions, and a possible weak chemical contribution for $\mathrm{Cu}(111)$. The balance between repulsive and attractive forces is achieved at different distances for $\mathrm{Cu}, \mathrm{Ag}$, and $\mathrm{Au}$ due to the different vdW radii of the metal atoms. However, similar adsorption energy for $\mathrm{Bz}$ on the three surfaces suggests the existence of universal binding energy trends for aromatic molecules on surfaces, resulting from a subtle balance between Pauli repulsion and many-body vdW attraction. In other words, for each surface the adsorption energy reaches its universal value, irrespective of the precise equilibrium position.

At this point, one might wonder about the transferability of the accuracy achieved by the DFT + MBD method if applied to more complex molecule-solid systems. Although general statements are difficult to make at the moment, preliminary calculations for more complex azobenzene $\left(\mathrm{C}_{12} \mathrm{H}_{10} \mathrm{~N}_{2}\right)$ and 3, 4, 9, 10-perylene-tetracarboxylic acid dianhydride $\left(\mathrm{C}_{24} \mathrm{O}_{6} \mathrm{H}_{8}\right)$ molecules on coinage metals yield similar findings, with DFT + MBD calculations resulting in vertical adsorption heights and binding energies within experimental error bars. A general conclusion that can nevertheless be drawn from our study is the crucial importance of treating both electronic exchange and correlation effects in a collective nonlocal fashion, beyond widely employed semilocal DFT functionals and simple pairwise corrections for vdW interactions. Obviously, further benchmarking to a level of theory that contains dispersion interactions and a multiconfigurational wave function nature of metals (beyond HSE + MBD) would be ideal.

In summary, we have carried out a systematic experimental and theoretical investigation of the structure and binding of benzene adsorbed on coinage metals. Our study provides benchmark values for adsorption properties of aromatic molecules under well-defined conditions. Remarkably, our experiments and calculations demonstrate that despite different electronic properties of $\mathrm{Cu}(111), \mathrm{Ag}(111)$, and $\mathrm{Au}(111)$ surfaces, the binding strength of benzene is equal on these three coinage metals. These initial results suggest the existence of universal binding energy trends for aromatic molecules on surfaces. Furthermore, we observed a significant renormalization of polarization of weakly interacting molecules or surface complexes, which provides an interesting research avenue for experimental studies of local properties of such hybrid interfaces. In addition, the importance of interface polarization grows with increasing molecular size and polarizability and may have a large impact on intermolecular interactions and assembly of molecular monolayers.

This research was supported by the European Research Council (ERC Starting Grant VDW-CMAT). The NIXSW experiments were carried out at the ID32 beam line of the European Synchrotron Radiation Facility (ESRF, Grenoble, France). We thank Julien Duvernay, Blanka Detlefs, Stephan Stremlau, and Erwan Varene for their assistance and experimental support.

*Corresponding author.

tkatchenko@fhi-berlin.mpg.de

[1] W. Lu and C. M. Lieber, Nat. Mater. 6, 841 (2007).

[2] F. Schedin, A. Geim, S. Morozov, E. Hill, P. Blake, M. Katsnelson, and K. Novoselov, Nat. Mater. 6, 652 (2007). 
[3] R. Waser and M. Aono, Nat. Mater. 6, 833 (2007).

[4] L. Kronik and N. Koch, MRS Bull. 35, 417 (2010).

[5] N. Koch, N. Ueno, and A. T. S. Wee, The Molecule-Metal Interface (John Wiley and Sons, New York, 2013).

[6] F. S. Tautz, Prog. Surf. Sci. 82, 479 (2007).

[7] N. Koch, A. Gerlach, S. Duhm, H. Glowatzki, G. Heimel, A. Vollmer, Y. Sakamoto, T. Suzuki, J. Zegenhagen, J. P. Rabe et al., J. Am. Chem. Soc. 130, 7300 (2008).

[8] Y. Xu, O. T. Hofmann, R. Schlesinger, S. Winkler, J. Frisch, J. Niederhausen, A. Vollmer, S. Blumstengel, F. Henneberger, N. Koch et al., Phys. Rev. Lett. 111, 226802 (2013).

[9] M. Willenbockel, D. Lüftner, B. Stadtmüller, G. Koller, C. Kumpf, S. Soubatch, P. Puschnig, M. G. Ramsey, and F. S. Tautz, Phys. Chem. Chem. Phys. 17, 1530 (2015).

[10] V. M. Hallmark and A. Campion, J. Chem. Phys. 84, 2933 (1986).

[11] P. Yannoulis, R. Dudde, K. Frank, and E. Koch, Surf. Sci. 189-190, 519 (1987).

[12] R. Dudde, K. H. Frank, and E. E. Koch, Surf. Sci. 225, 267 (1990).

[13] K. H. Frank, P. Yannoulis, R. Dudde, and E. E. Koch, J. Chem. Phys. 89, 7569 (1988).

[14] P. Avouris and J. E. Demuth, J. Chem. Phys. 75, 4783 (1981).

[15] X.-L. Zhou, M. Castro, and J. White, Surf. Sci. 238, 215 (1990).

[16] T. J. Rockey, M. Yang, and H.-L. Dai, J. Phys. Chem. B 110, 19973 (2006).

[17] V. G. Ruiz, W. Liu, E. Zojer, M. Scheffler, and A. Tkatchenko, Phys. Rev. Lett. 108, 146103 (2012).

[18] W. Liu, V. G. Ruiz, G.-X. Zhang, B. Santra, X. Ren, M. Scheffler, and A. Tkatchenko, New J. Phys. 15, 053046 (2013).

[19] N. Almora-Barrios, G. Carchini, P. Błonski, and N. Lopez, J. Chem. Theory Comput. 10, 5002 (2014).

[20] W. Liu, A. Tkatchenko, and M. Scheffler, Acc. Chem. Res. 47, 3369 (2014).

[21] J. Zegenhagen, Surf. Sci. Rep. 18, 202 (1993).

[22] D. Woodruff, Prog. Surf. Sci. 57, 1 (1998).

[23] G. Mercurio, R. Maurer, W. Liu, S. Hagen, F. Leyssner, P. Tegeder, J. Meyer, A. Tkatchenko, S. Soubatch, K. Reuter et al., Phys. Rev. B 88, 035421 (2013).

[24] G. Mercurio, O. Bauer, M. Willenbockel, N. Fairley, W. Reckien, C. H. Schmitz, B. Fiedler, S. Soubatch, T. Bredow, M. Sokolowski et al., Phys. Rev. B 87, 045421 (2013).
[25] G. Mercurio, Study of Molecule-Metal Interfaces by Means of the Normal Incidence X-ray Standing Wave Technique, Schriften des Forschungszentrum Jülich Vol. 49 (Diss., RWTH Aachen, University, 2012), http://www.fz-juelich .de/zb/juwel.

[26] Torricelli is an XSW data analysis and simulation program written by G. Mercurio; copies can be obtained from s.tautz@fz-juelich.de.

[27] J. W. Niemantsverdriet, P. Dolle, K. Markert, and K. Wandelt, J. Vac. Sci. Technol. A 5, 875 (1987).

[28] D. L. S. Nieskens, A. P. van Bavel, and J. W. Niemantsverdriet, Surf. Sci. 546, 159 (2003).

[29] D. A. King, Surf. Sci. 47, 384 (1975).

[30] J. T. Stuckless, N. Al-Sarraf, C. Wartnaby, and D. A. King, J. Chem. Phys. 99, 2202 (1993).

[31] J. T. Stuckless, N. A. Frei, and C. T. Campbell, Rev. Sci. Instrum. 69, 2427 (1998).

[32] J. Yang, W. Hu, D. Usvyat, D. Matthews, M. Schütz, and G. K.-L. Chan, Science 345, 640 (2014).

[33] A. Tkatchenko, L. Romaner, O. T. Hofmann, E. Zojer, C. Ambrosch-Draxl, and M. Scheffler, MRS Bull. 35, 435 (2010).

[34] J. B. Neaton, M. S. Hybertsen, and S. G. Louie, Phys. Rev. Lett. 97, 216405 (2006).

[35] J. Heyd, G. E. Scuseria, and M. Ernzerhof, J. Chem. Phys. 118, 8207 (2003).

[36] J. Heyd, G. E. Scuseria, and M. Ernzerhof, J. Chem. Phys. 124, 219906 (2006).

[37] A. Tkatchenko, R. A. DiStasio, Jr., R. Car, and M. Scheffler, Phys. Rev. Lett. 108, 236402 (2012).

[38] A. Ambrosetti, A. M. Reilly, R. A. DiStasio, Jr., and A. Tkatchenko, J. Chem. Phys. 140, 18A508 (2014).

[39] V. Blum, R. Gehrke, F. Hanke, P. Havu, V. Havu, X. Ren, K. Reuter, and M. Scheffler, Comput. Phys. Commun. 180, 2175 (2009).

[40] See Supplemental Material at http://link.aps.org/ supplemental/10.1103/PhysRevLett.115.036104 for a technical description of NIXSW and TPD experiments, as well as DFT calculations.

[41] E. van Lenthe, E.-J. Baerends, and J. G. Snijders, J. Chem. Phys. 101, 9783 (1994).

[42] J. P. Perdew, K. Burke, and M. Ernzerhof, Phys. Rev. Lett. 77, 3865 (1996). 